01

\title{
Сверхкритический резонанс в присутствии сильного магнитного поля
}

\author{
(С) Д.А. Тумаков ${ }^{1,2}$, И.А. Мальцев ${ }^{1}$, Р.В. Попов ${ }^{1}$, В.М. Шабаев ${ }^{1}$ \\ ${ }^{1}$ Санкт-Петербургский государственный университет, \\ 199034 Санкт-Петербург, Россия \\ ${ }^{2}$ ФТИ им. А.Ф. Иоффре РАН, \\ 194021 Санкт-Петербург, Россия \\ e-mail: d.tumakov@spbu.ru, i.maltsev@spbu.ru
}

Поступила в редакцию 27.12.2021 г.

В окончательной редакции 27.12.2021 г.

Принята к публикации 10.01.2022 г.

Рассчитаны положение и ширина сверхкритического резонанса, образующегося при погружении основного состояния одноэлектронного иона в отрицательно-энергетический континуум в присутствии сильного магнитного поля. Исследовано влияние величины магнитного поля на параметры резонанса.

Ключевые слова: сверхкритическое поле, комплексное вращение.

DOI: 10.21883/OS.2022.04.52279.3089-21

\section{Введение}

Квантовая электродинамика предсказывает, что в сверхсильном кулоновском поле должны наблюдаться качественно новые эффекты, вызванные нестабильностью вакуума [1-6]. В частности, как было показано в работах $[2,3]$, при заряде ядра, превышающем критическое значение $Z_{\mathrm{cr}} \gtrsim 173$, основное состояние водородоподобного иона погружается в отрицательный континуум, становясь резонансным состоянием. В результате с вероятностью, определяемой шириной резонанса, вакуум распадается с образованием позитрона. Несмотря на то, что ядер с зарядом, близким к критическому, не существует, в недавних теоретических работах было продемонстрировано, что спонтанный распад вакуума можно наблюдать в экспериментах с медленными столкновениями тяжелых ионов, совокупный заряд которых превышает критический $[7,8]$. Однако подобное наблюдение представляют собой сложную задачу с точки зрения эксперимента. Поэтому целесообразно рассмотреть возможность увеличения ширины сверхкритического резонанса, а значит и вероятности распада вакуума, с помощью дополнительного внешнего поля.

В данной работе рассматривается поведение основного состояния водородоподобного иона, помещенного в сверхсильное однородное магнитное поле. Как было показано в работах $[9,10]$, наличие такого поля эффективно уменьшает критическое значение заряда ядра $Z_{\mathrm{cr}}$, при котором основное состояние погружается в отрицательноэнергетический континуум. Это объясняется тем, что волновая функция электрона в основном состоянии „сжимается“, становясь квазиодномерной, что усиливает сингулярность кулоновского потенциала. По этой же причине можно ожидать, что присутствие магнитного поля увеличит вероятность спонтанного распада вакуума. Основной целью представленной работы является исследование данного эффекта.
Ранее в работах [9-12] исследовалась зависимость $Z_{\text {cr }}$ от напряженности однородного магнитного поля. Кроме того, проводились вычисления энергии основного электронного состояния, но только вплоть до его погружения в отрицательно-энергетический континуум и превращения в резонанс. Необходимо отметить, что в работах $[11,12]$ было также рассмотрено влияние экранировки кулоновского поля ядра, возникающей в сверхсильном магнитном поле, на значение критического заряда.

В представленной работе используется метод комплексного вращения, который позволяет получить параметры резонансных состояний. Данный метод основан на повороте радиальной координаты в комплексную плоскость. В результате волновые функции резонансов становятся квадратично интегрируемыми, а соответствующие энергии принимают комплексные значения, действительная часть которых определяет положение резонанса, а мнимая - ширину. В работах [13-16] техника комплексного вращения успешно использовалась для расчета параметров сверхкритического резонанса в кулоновском поле. Подробное описание метода комплексного вращения и его многочисленных приложений можно найти в обзорах $[17,18]$. В данной работе с помощью техники комплексного вращения получена энергии основного состояния одноэлектронного иона после погружения в отрицательно-энергетический континуум в присутствии магнитного поля. Также была вычислена ширина сверхкритического резонанса в зависимости от величины магнитного поля, что позволило оценить его влияние на вероятность спонтанного распада вакуума.

В работе используется атомная система единиц: $\hbar=1$, $m=1, e=-1$. Скорость света в вакууме в таких единицах равна $1 / \alpha \approx 137.036$, где $\alpha-$ постоянная тонкой структуры. Величина напряженности магнитного поля в результатах расчетов приводится в единицах 
критической напряженности $B_{0}=\frac{m^{2} c^{3}}{\hbar|e|}$, которая связана с единицами СИ как $c^{-1} B_{0} \approx 4.41 \cdot 10^{9} \mathrm{~T}$.

\section{Теория и методы расчета}

В присутствии постоянного внешнего магнитного поля волновая функция электрона в водородоподобной системе удовлетворяет стационарному уравнению Дирака:

$$
H_{0} \Psi(\mathbf{r})=E \Psi(\mathbf{r})
$$

где гамильтониан

$$
\begin{aligned}
H_{0} & =c \boldsymbol{\alpha} \cdot\left(\mathbf{p}+\frac{\mathbf{A}}{c}\right)+c^{2} \beta+V_{\mathrm{nucl}} \\
& =c \boldsymbol{\alpha} \cdot \mathbf{p}+c^{2} \beta+V_{\mathrm{nucl}}+V_{\mathrm{ext}} .
\end{aligned}
$$

Здесь $c \approx 137$ - скорость света в вакууме, $\alpha$ и $\beta-$ матрицы Дирака. Сферически симметричный потенциал $V_{\text {nucl }}(r)$ описывает взаимодействие с ядром. Если явно не указано иное, то в расчетах используется модель равномерно заряженного шара:

$$
V_{\text {nucl }}(r)= \begin{cases}-\frac{Z}{r}, & r \geqslant R_{\text {nucl }} \\ -\frac{Z}{2 r}\left(3-\frac{r^{2}}{R_{\text {nucl }}}\right), & r<R_{\text {nucl }}\end{cases}
$$

Потенциал $V_{\text {ext }}(\mathbf{r})$ описывает взаимодействие электрона с постоянным магнитным полем, направленным вдоль оси z. Выберем векторный потенциал в виде $\mathbf{A}=\frac{1}{2}[\mathbf{B} \times \mathbf{r}]$, тогда

$$
V_{\mathrm{ext}}(\mathbf{r})=\frac{1}{2} \mathbf{B} \cdot[\mathbf{r} \times \boldsymbol{\alpha}] .
$$

Рассмотрим решение уравнения Дирака (1) в сферической системе координат $(r, \theta, \varphi)$. Ввиду аксиальной симметрии гамильтониана (2) проекция $m_{j}=-1 / 2$ полного момента электрона на ось $z$ будет постоянной, и решение можно искать в следующем виде:

$$
\Psi(r, \theta, \varphi)=\frac{1}{\sqrt{2 \pi} r}\left(\begin{array}{c}
\mathrm{e}^{-i \varphi} \psi_{1}(r, \theta) \\
\psi_{2}(r, \theta) \\
i \mathrm{e}^{-i \varphi} \psi_{3}(r, \theta) \\
i \psi_{4}(r, \theta)
\end{array}\right)
$$

Четырехкомпонентная функция $\psi(r, \theta)$, составленная из скалярных функций $\psi_{i}(r, \theta)$, удовлетворяет следующему уравнению:

$$
\widetilde{H} \psi(r, \theta)=E \psi(r, \theta),
$$

где

$$
\widetilde{H}=\left(\begin{array}{cc}
\left(V_{\text {nucl }}+c^{2}\right) \cdot \mathbf{1}_{2} & c D \\
-c D & \left(V_{\text {nucl }}-c^{2}\right) \cdot \mathbf{1}_{2}
\end{array}\right)
$$

и

$$
\begin{aligned}
D= & \frac{1}{2 c} B r \sin \theta i \sigma_{y}+\left(\sigma_{x} \sin \theta+\sigma_{z} \cos \theta\right)\left(\frac{\partial}{\partial r}-\frac{1}{r}\right) \\
& +\frac{1}{r}\left(\sigma_{x} \cos \theta-\sigma_{z} \sin \theta\right) \frac{\partial}{\partial \theta}+\frac{1}{2 r \sin \theta}\left(\sigma_{x}-i \sigma_{y}\right) .
\end{aligned}
$$

Здесь $\sigma_{x}, \sigma_{y}$ и $\sigma_{z}-$ матрицы Паули, $\mathbf{1}_{2}-$ единичная матрица $2 \cdot 2$.

Для поиска резонансных решений уравнения (6) в данной работе применялся метод комплексного вращения, в рамках которого радиальная координата $r$,поворачивается“ на угол $\Theta(r)$ в комплексную плоскость:

$$
\tilde{r}=r e^{i \Theta(r)} .
$$

Зависимость угла $\Theta$ от значения $r$ позволяет осуществлять поворот как всей оси, так и ее части. Использование различных параметров, определяющих вид функции $\Theta(r)$, позволяет улучшать сходимость получаемых результатов.

Гамильтониан $\widetilde{H}$ после поворота уже не является эрмитовым, и энергия $E$ уровня принимает комплексные значения. Удобно представить ее в виде

$$
E=\varepsilon+i \Gamma / 2,
$$

где $\varepsilon-$ положение резонанса, а $Г-$ его ширина. Уравнение (6) может быть дискретизировано с помощью обобщенного псевдоспектрального метода [19,20], в рамках которого оно сводится к задаче на поиск собственных значений комплекснозначной матрицы. В наших расчетах мы используем библиотеку FEAST [21], позволяющую эффективно находить собственные значения разреженной матрицы, лежащие внутри заданного контура в комплексной плоскости.

\section{Результаты и обсуждение}

С целью проверки работы метода, изложенного в предыдущем разделе, был проведен расчет энергии основного состояния водородоподобного иона свинца $(Z=82)$, помещенного в однородное магнитное поле, для разных значений магнитного поля $B$. Результаты представлены на рисунке в сравнении с соответствующими значениями из работы [10]. Для сравнения результатов с прежними в данном случае использовалась точечная модель ядра. Из графика видно, что полученные результаты хорошо согласуются с соответствующими значениями из статьи [10]. При этом поле порядка $10^{11}$ Т переводит данный ион в сверхкритический режим (т.е. происходит погружение основного состояния в отрицательно-энергетический континуум). В представленной работе с помощью метода комплексного вращения удалось отследить положение основного состояния и после погружения, что также отображено на графике.

В качестве примера системы, которая является сверхкритической даже в отсутствие магнитного поля, был выбран гипотетический ион с зарядом ядра $Z=178$. При таком заряде ядра основное состояние и без внешнего поля уже встроено в отрицательно-энергетический континуум и представляет собой резонанс с некоторой ненулевой шириной. С целью изучить влияние магнитного поля были проведены расчеты положения и ширины 
Положение и ширина резонансного состояния $1 s_{-1 / 2}$ водородоподобного иона с $Z=178$, помещенного в однородное магнитное поле индукции $B\left(c^{-1} B_{0} \approx 4.41 \cdot 10^{9} \mathrm{~T}\right)$

\begin{tabular}{c|c|c}
\hline$B / B_{0}$ & $\varepsilon / c^{2}$ & $\Gamma / c^{2}$ \\
\hline 0 & -1.563 & $3.4 \cdot 10^{-3}$ \\
0.053 & -1.566 & $3.7 \cdot 10^{-3}$ \\
0.1 & -1.569 & $4.0 \cdot 10^{-3}$ \\
0.266 & -1.578 & $4.4 \cdot 10^{-3}$ \\
0.4 & -1.587 & $4.7 \cdot 10^{-3}$
\end{tabular}

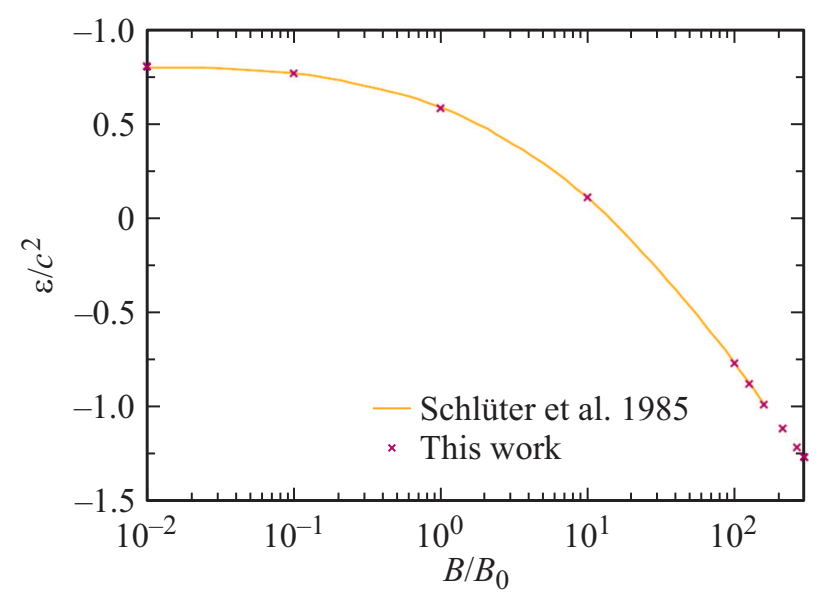

Вещественная часть энергии состояния $1 s_{-1 / 2}$ водородоподобного иона свинца $(Z=82)$ в зависимости от индукции $B$ однородного магнитного поля $\left(c^{-1} B_{0} \approx 4.41 \cdot 10^{9} \mathrm{~T}\right)$.

сверхкритического резонанса для нескольких значений величины поля $B$. Результаты представлены в таблице.

Из результатов видно, что положение резонанса сдвигается вниз с усилением поля. При этом ширина уровня также возрастает, что означает увеличение вероятности спонтанного распада вакуума. Существенное изменение этой вероятности происходит при величине магнитного поля $B$, значительно меньше критической $B_{0}$. Несмотря на то, что рассмотренные значения $B$ далеки от доступных экспериментально, полученные результаты указывают на возможность усиления сигнала от спонтанного распада вакуума в сверхкритических столкновениях тяжелых ионов в присутствии магнитного поля.

\section{Заключение}

Выполнен расчет положения и ширины основного состояния сверхкритического одноэлектронного иона в присутствии однородного магнитного поля. Расчет проведен с помощью метода комплексного вращения с использованием конечного базисного набора. Продемонстрировано увеличение вероятности спонтанного распада вакуума в присутствии магнитного поля.

\section{Финансирование работы}

Исследования проведены при финансовой поддержке гранта Президента Российской Федерации No MK1626.2020.2, а также при финансовой поддержке РФФИ и Госкорпорации „Росатом“ в рамках научного проекта № 20-21-00098. Работа И.А.М. также была поддержана фондом развития теоретической физики и математики „БАЗИС“. Расчеты были проведены на оборудовании „Вычислительного центра СПбГУ“.

\section{Конфликт интересов}

Авторы заявляют, что у них нет конфликта интересов.

\section{Список литературы}

[1] I. Pomeranchuk, J. Smorodinsky. J. Phys. USSR, 9, 97 (1945).

[2] С.С. Герштейн, Я.Б. Зельдович. ЖЭТФ, 57, 654 (1969). [S.S. Gershtein, Y.B. Zel'dovich. Sov. Phys. JETP, 30, 358 (1970)].

[3] W. Pieper, W. Greiner. Z. Phys., 218, 327 (1969). DOI: $10.1007 / \mathrm{BF} 01670014$

[4] Я.Б. Зельдович, В.С. Попов. УФН, 105, 403 (1971). DOI: 10.3367/UFNr.0105.197111b.0403 [Y.B. Zeldovich, V.S. Popov. Sov. Phys. Usp., 14, 673 (1972). DOI: 10.1070/PU1972v014n06ABEH004735].

[5] W. Greiner, B. Müller, J. Rafelski. Quantum Electrodynamics of Strong Fields, 1st ed. (Springer-Verlag, Berlin, 1985). DOI: $10.1007 / 978-3-642-82272-8$

[6] U. Müller-Nehler, G. Soff. Phys. Rep., 246, 101 (1994). DOI: 10.1016/0370-1573(94)90068-X

[7] I.A. Maltsev, V.M. Shabaev, R.V. Popov, Y.S. Kozhedub, G. Plunien, X. Ma, Th. Stöhlker, D.A. Tumakov. Phys. Rev. Lett., 123, 113401 (2019). DOI: 10.1103/PhysRevLett.123.113401

[8] R.V. Popov, V.M. Shabaev, D.A. Telnov, I.I. Tupitsyn, I.A. Maltsev, Y.S. Kozhedub, A.I. Bondarev, N.V. Kozin, X. Ma, G. Plunien, T. Stöhlker, D.A. Tumakov, V.A. Zaytsev. Phys. Rev. D, 102, 07600 (2020). DOI: 10.1103/PhysRevD.102.076005

[9] В.Н. Ораевский, А.И. Рез, В.Б. Семикоз. ЖЭТФ, 72(3), 820 (1977). [V.N. Oraevskii, A.I. Rex, V.B. Semikoz. Sov. Phys. JETP, 45, 428 (1977).].

[10] P. Schlüter, G. Soff., K.H. Wietschorke, W. Greiner. J. Phys. B, 18, 1685 (1985). DOI: 10.1088/0022-3700/18/9/005

[11] S.I. Godunov, B. Machet, M.I. Vysotsky. Phys. Rev. D, 85, 044058 (2012). DOI: 10.1103/PhysRevD.85.044058

[12] М.И. Высоцкий, С.И. Годунов. УФН, 184, 206 (2014). DOI: $10.3367 /$ UFNr.0184.201402j.0206 [M.I. Vysotskii, S.I. Godunov. Phys. Usp., 57, 194 (2014). DOI: 10.3367/UFNe.0184.201402j.0206].

[13] E. Ackad, M. Horbatsch. Phys. Rev. A, 75, 022508 (2007). DOI: $10.1103 /$ PhysRevA.75.022508

[14] E. Ackad, M. Horbatsch. Phys. Rev. A, 76, 022503 (2007). DOI: 10.1103/PhysRevA.76.022503

[15] A. Marsman, M. Horbatsch. Phys. Rev. A, 84, 032517 (2011). DOI: $10.1103 /$ PhysRevA.84.032517 
[16] И.А. Мальцев, В.М. Шабаев, В.А. Зайцев. Р.В. Попов, Д.А. Тумаков. Опт. и спектр., 128(8), 1094 (2020).

DOI: $10.21883 /$ OS.2020.08.49703.117-20 [I.A. Maltsev, V.M. Shabaev, V.A. Zaytsev, R.V. Popov, Y.S. Kozhedub, D.A. Tumakov. Opt. Spectrosc., 128, 1100 (2020). DOI: $10.1134 / \mathrm{S} 0030400 \mathrm{X} 2008024 \mathrm{X}$.

[17] N. Moiseyev. Phys. Rep., 302, 212 (1998). DOI: $10.1016 / \mathrm{S} 0370-1573(98) 00002-7$

[18] E. Lindroth, L. Argenti. Adv. Quantum Chem., 63, 247 (2012). DOI: 10.1016/B978-0-12-397009-1.00005-9

[19] G. Yao, S.-I. Chu. Chem. Phys. Lett., 204, 381 (1993). DOI: 10.1016/0009-2614(93)90025-V

[20] D.A. Telnov, S.-I. Chu. Phys. Rev. A, 59, 2864 (1999). DOI: 10.1103/PhysRevA.59.2864

[21] E. Polizzi. Phys. Rev. B, 79, 115112 (2009).

DOI: 10.1103/PhysRevB.79.115112 